Check for updates

Cite this: RSC Adv., 2017, 7, 53422

Received 6th October 2017

Accepted 31st October 2017

DOI: 10.1039/c7ra11011a

rsc.li/rsc-advances

\title{
Sodium-alginate biopolymer as a template for the synthesis of nontoxic red emitting $\mathrm{Mn}^{2+}$-doped $\mathrm{CdS}$ nanoparticles $\uparrow$
}

\author{
M. Kuzmanović, (D) ab D. K. Božanić, ${ }^{\text {bc }}$ D. Milivojević, ${ }^{c}$ D. Mitić Ćulafić, ${ }^{d}$ S. Stanković, ${ }^{d}$ \\ C. Ballesteros ${ }^{e}$ and J. Gonzalez-Benito (D) *b
}

\begin{abstract}
Manganese-doped cadmium sulfide (CdS:Mn) nanoparticles were prepared by chemical synthesis using sodium-alginate as template. The preparation of the nanocomposites involved ionic crosslinking of the biopolymer by dimerization of its $\alpha$-L-guluronic monomers with $\mathrm{Cd}^{2+}$ and subsequent formation of the semiconductor nanoparticles upon addition of sulfide ions in the presence of $\mathrm{Mn}^{2+}$. The crystalline phase of CdS in the material was confirmed by XRD. The surface morphology of the nanocomposites was investigated by SEM. The observation by TEM showed that the CdS:Mn particles were spherical in shape with diameters of approximately $4 \mathrm{~nm}$. EPR measurements of the CdS:Mn-alginate nanocomposite showed that the $\mathrm{Mn}^{2+}$ ions were incorporated in cationic sites of CdS with lower symmetry. Due to a distorted crystal field induced by the $\mathrm{Mn}^{2+}$ ions, photoluminescence spectra of the CdS:Mn-alginate showed red fluorescence between $650 \mathrm{~nm}$ and $750 \mathrm{~nm}$ falling into the optical window for bioimaging in which the light has its maximum tissue penetration depth. It was demonstrated that the interaction between the nanoparticles and the matrix prevents release of CdS into the environment, leading to low acute toxicity of the nanocomposites.
\end{abstract}

\section{Introduction}

An important part of modern nanoscience and nanotechnology is the investigation of new materials composed of a polymer matrix filled with semiconductor nanoparticles., ${ }^{\mathbf{1} 2}$ These types of nanocomposites combine characteristic optical, electrical, magnetic or catalytic properties of semiconductors with the chemical stability and processability of polymers. Apart from representing good host matrices, polymers can also act as templates to control semiconductor nanoparticle growth and surface properties. These specific features have inspired investigations directed towards disclosure of possible applications of semiconductor-polymer nanocomposites..$^{3-5}$ An ongoing

${ }^{a}$ Department of Material Science and Engineering, Faculty of Engineering and Architecture, Ghent University, Technologiepark 915, 9052 Zwijnaarde, Belgium

${ }^{b}$ Department of Materials Science and Engineering and Chemical Engineering, IQMAAB, Universidad Carlos III de Madrid, Avenida de la Universidad 30, 28911 Leganés (Madrid), Spain. E-mail: javid@ing.uc3m.es; Fax: +34 916249430; Tel: +34 916248870

'University of Belgrade, Vinča Institute of Nuclear Sciences, P.O. Box 522, 11001 Belgrade, Serbia. E-mail: bozanic@vinca.rs

${ }^{d}$ University of Belgrade, Faculty of Biology, Center for Genotoxicology and Ecogenotoxicology, Studentski trg 16, 11001 Belgrade, Serbia

${ }^{e}$ Department of Physics, Universidad Carlos III de Madrid, Avenida de la Universidad 30, 28911 Leganés (Madrid), Spain

† Electronic supplementary information (ESI) available. See DOI: 10.1039/c7ra11011a endeavor of fabrication of semiconductor nanostructures for biomedical applications has led to the development of procedures that employ polysaccharides, such as starch, cellulose, chitosan, alginate etc., as matrices for encapsulation of semiconductor nanoparticles. ${ }^{6-14}$ In addition to being highly abundant, biocompatible and biodegradable, these biopolymers have specific macromolecular structure that presents a good environment for the controlled growth of metallic and semiconductor nanoparticles.

Alginic acid or alginate, a macromolecule extracted from brown marine algae, is a block-copolymer of $(1,4)$-linked $\beta$-Dmannuronic and $\alpha$-L-guluronic (G) acids. ${ }^{15}$ The key property of alginate regarding its application as a template for the controlled growth and stabilization of nanostructures is its affinity for divalent metals. ${ }^{\mathbf{1 6}}$ The interaction of alginate with divalent cations takes place via carboxylate groups of $\alpha$-Lguluronic monomers that results in dimerization of $\alpha$-L-guluronic block sequences and ordered, e.g. "egg-box", conformation of the biopolymer. Due to this ionic cross-linking the diffusion of cations throughout the matrix is limited that, in the case of nanoparticle formation during in situ chemical syntheses, results in narrow size distribution of the resulting nanostructures and good dispersion of the inorganic phase in the matrix. Besides, as a consequence of the change in conformation of alginate high concentrations of the nanoparticles relative to the polymer can be achieved. Moreover, due to its amphiphilic nature, alginate can be employed to obtain 
biocompatible and water-soluble quantum dots via phase transfer, as it was previously shown by Wang et al. in the case of CdSe/ZnS nanoparticles. ${ }^{17}$ This polysaccharide was already used as an environment for controlled growth of II-VI semiconductors, ${ }^{18} \mathrm{Co}$, Ni, and CoNi nanoparticles, ${ }^{19}$ iron oxide nanostructures ${ }^{20}$ as well as antimicrobial $\mathrm{ZnO}$ nanoparticles ${ }^{21,22}$ and hybrid $\mathrm{ZnO} / \mathrm{Ag}$ nanocubes. ${ }^{23}$ However, to the best of our knowledge, alginates were not used for the preparation of diluted magnetic semiconductor nanoparticles such as the CdS:Mn described in the present work. In this study, we used an in situ chemical synthesis for the preparation of CdS:Mnalginate nanocomposites in which the nanoparticles were formed directly within the biopolymer matrix.

Cadmium sulfide is an n-type II-VI semiconductor with a direct band gap of $\sim 2.4 \mathrm{eV}$ and an exciton Bohr radius, $a_{\mathrm{b}}$, of $\sim 2.8 \mathrm{~nm} .{ }^{24}$ Furthermore, due to size-dependent luminescence in the visible range, CdS nanostructures have found application in optoelectronics and photoconductive devices,4 or as a core component in core-shell quantum dots for biomedical imaging. ${ }^{5}$ Photoluminescent properties of CdS nanoparticles, as well as quantum dots in general, are very sensitive to their environment, as well as to surface characteristics of the nanoparticle, because of the presence of surface defects such as sulfur vacancies or sulfur dangling bonds. ${ }^{25}$ Efficient modification of optical properties of CdS nanomaterials can be achieved by doping of the semiconductor with transition metal ions $\left(\mathrm{Cu}^{2+}, \mathrm{Fe}^{2+}, \mathrm{Mn}^{2+}\right.$ etc.). This way, discrete energy states, formed by splitting of the ionic levels under the influence of the crystal field of the host's lattice, are being introduced into the band gap of the semiconductor. ${ }^{26}$ The excitation/emission processes from these atomic-like states are usually very efficient and the photoluminescence of the dopant usually dominates the exciton photoemission from the host. ${ }^{27,28}$ Furthermore, a complete suppression of the emission from surface states of CdS upon doping with $\mathrm{Mn}^{2+}$ ions was reported. ${ }^{27}$ For bioimaging applications in particular, the photoemission from atomic-like states of doped quantum dots provide additional advantages in comparison to exciton luminescence such as negligible self-quenching, substantial Stokes shift and large photoluminescence lifetime. ${ }^{29}$

In the case of $\mathrm{Mn}^{2+}$ doped wide band gap II-VI semiconductors, the emission originates from localized $3 \mathrm{~d}$ states that are strongly affected by the crystal field of the lattice site occupied by the ion. ${ }^{30,31}$ For $\mathrm{Mn}^{2+}$ positioned in sites with tetrahedral coordination, spin-sextet ${ }^{6} \mathrm{~S}$ ground state and spinquartet ${ }^{4} \mathrm{G},{ }^{4} \mathrm{P},{ }^{4} \mathrm{D}$, and ${ }^{4} \mathrm{~F}$ excited states of free ion split into ${ }^{6} \mathrm{~A}_{1}\left({ }^{6} \mathrm{~S}\right),{ }^{4} \mathrm{~T}_{1}\left({ }^{4} \mathrm{G}\right),{ }^{4} \mathrm{~T}_{2}\left({ }^{4} \mathrm{G}\right),{ }^{4} \mathrm{E}\left({ }^{4} \mathrm{G}\right),{ }^{4} \mathrm{~A}_{1}\left({ }^{4} \mathrm{G}\right),{ }^{4} \mathrm{E}\left({ }^{4} \mathrm{D}\right)$, and ${ }^{4} \mathrm{~T}_{2}\left({ }^{4} \mathrm{D}\right)$ energy levels under the influence of the crystal field. ${ }^{26,32}$ The transitions between the energy levels in free $\mathrm{Mn}^{2+}$ ions are spin forbidden and inefficient. On the other hand, upon light absorption, electrons from the ${ }^{6} \mathrm{~A}_{1}\left({ }^{6} \mathrm{~S}\right)$ state are being transferred to ${ }^{4} E\left({ }^{4} G\right)$ and ${ }^{4} A_{1}\left({ }^{4} G\right)$ energy states and, subsequently recombine the first excited ${ }^{4} \mathrm{~T}_{1}\left({ }^{4} \mathrm{G}\right)$ state by nonradiative transitions. ${ }^{33}$ Finally, transitions between the ${ }^{4} \mathrm{~T}_{1}\left({ }^{4} \mathrm{G}\right)$ state and the lowest ${ }^{6} \mathrm{~A}_{1}\left({ }^{6} \mathrm{~S}\right)$ state take place resulting in a typical $2.12 \mathrm{eV}$ (585 nm) yellow emission of $\mathrm{Mn}^{2+}$ in the semiconductor host. ${ }^{27}$ Contrary to II-VI semiconductor nanostructures, the photoluminescence of $\mathrm{Mn}^{2+}$ is not affected significantly by the change in size of the particles and it does not depend significantly on the composition of the nanostructured host. ${ }^{34,35}$ However, it does depend on the crystal field strength since, in the case of tetrahedral coordination, the increased splitting of the triplet ${ }^{4} \mathrm{~T}_{1}\left({ }^{4} \mathrm{G}\right)$ state would lead to decrease in the energy difference between ${ }^{4} \mathrm{~T}_{1}\left({ }^{4} \mathrm{G}\right)$ and ${ }^{6} \mathrm{~A}_{1}\left({ }^{6} \mathrm{~S}\right)$ state and, consequently, to the red shift of the emission. ${ }^{36,37}$ The effect of the crystal field strength, however, on the position of the photoluminescence band of $\mathrm{Mn}$ doped semiconductor nanostructures is very small, ${ }^{30}$ whereas the energy of emitted photons is affected to a greater extent by the changes in the crystal field symmetry. Sarma and coauthors ${ }^{35}$ showed that luminescence of $\mathrm{Mn}^{2+}$ doped $\mathrm{ZnS}-\mathrm{CdS}$ alloy nanocrystals can be tuned in the wide range between $1.9 \mathrm{eV}$ and $2.4 \mathrm{eV}$, as a consequence of the availability of different lattice sites with different crystal fields that $\mathrm{Mn}^{2+}$ ions can occupy.

In this article, we report on structural and optical characteristics of manganese doped cadmium sulfide nanoparticles prepared in aqueous solution by an in situ chemical synthesis using biocompatible Na-alginate biopolymer as a template. It will be studied how the distorted crystal field surrounding the $\mathrm{Mn}^{2+}$ ions affects the CdS:Mn-alginate nanocomposites photoluminescence regarding their potential use in bioimaging, since particular photoluminescent characteristics of the nanocomposites coupled with the biocompatibility of alginate open up a possibility of application of the prepared materials as fluorescent substrates for tissue imaging in the NIR domain. ${ }^{38}$

\section{Experimental}

\subsection{Materials}

The following materials were purchased from Sigma-Aldrich Chemical Co. and used as received: alginic acid sodium salt from brown algae (Na-alginate), cadmium acetate $\left(\mathrm{Cd}\left(\mathrm{CH}_{3}\right.\right.$ $\left.\mathrm{COO})_{2}\right)$, thioacetamide $\left(\mathrm{C}_{2} \mathrm{H}_{5} \mathrm{NS}\right)$, and manganese(II) nitrate tetrahydrate $\left(\mathrm{Mn}\left(\mathrm{NO}_{3}\right)_{2}\right)$. High purity water was used as a solvent in all synthesis steps.

\subsection{Preparation of the CdS-alginate and CdS:Mn-alginate nanocomposites}

Batch aqueous solutions of Na-alginate (1 wt/vol\%), $\mathrm{Cd}\left(\mathrm{CH}_{3}\right.$ $\mathrm{COO})_{2}(0.1 \mathrm{M}), \mathrm{C}_{2} \mathrm{H}_{5} \mathrm{NS}(0.1 \mathrm{M})$, and $\mathrm{Mn}\left(\mathrm{NO}_{3}\right)_{2}(0.1 \mathrm{M})$ were prepared shortly before the synthesis of the nanocomposites. To remove the residual oxygen the solutions were purged by nitrogen for $30 \mathrm{~min}$ prior to the synthesis of the nanocomposites.

To prepare CdS-alginate and CdS:Mn-alginate nanocomposites, $25 \mathrm{~mL}$ of the Na-alginate solution was added drop by drop to $50 \mathrm{~mL}$ of $0.1 \mathrm{M}$ of $\mathrm{Cd}\left(\mathrm{CH}_{3} \mathrm{COO}\right)_{2}$ solution mixed previously with $0 \mathrm{~mL}, 0.5 \mathrm{~mL}, 1 \mathrm{~mL}$, or $2 \mathrm{~mL}$ of $0.1 \mathrm{M} \mathrm{Mn}\left(\mathrm{NO}_{3}\right)_{2}$ solution subjected to a continuous flow of nitrogen. Due to the presence of $\mathrm{Cd}^{2+}$ ions, the biopolymer underwent the process of ionic cross-linking, which was manifested by the formation of a white solid precipitate in the reactor flask. The cross-linked polymer was collected and purified by 3 times centrifugation (3000 rpm for 5 minutes) and subsequent re-dispersion. After that, the purified cadmium cross-linked alginate (Cd-alginate) 
biopolymer was mixed with $50 \mathrm{~mL}$ of water and heated to $80{ }^{\circ} \mathrm{C}$ under constant stirring. While heating, $50 \mathrm{~mL}$ of the $\mathrm{C}_{2} \mathrm{H}_{5} \mathrm{NS}$ was added drop by drop to the dispersion. The cross-linked polymer pellets changed their color to yellow upon the addition of the sulfide source indicating the formation of the CdS:Mn nanoparticles. The reaction was carried out for $1 \mathrm{~h}$ under the same conditions, after which the dispersion was cooled to room temperature and centrifuged 3 times $(3000 \mathrm{rpm}$, $15 \mathrm{~min}$ ) in water and ethanol to separate the precipitate and remove residual chemicals. Finally, the nanocomposites were dried at ambient conditions and pulverized using a mortar. The estimated composition of the nanoparticles with respect to the biopolymer was $10 \mathrm{wt} \% \mathrm{CdS}: \mathrm{Mn}$.

\subsection{Characterization of the CdS-alginate and CdS:Mn- alginate nanocomposites}

The X-ray diffraction (XRD) measurements were performed on a Philips X-PERT instrument using the $\mathrm{K} \alpha_{1 / 2}$ doublet lines of copper (wavelength $\lambda=0.154 \mathrm{~nm}$ ).

The surface morphology of the CdS-alginate and CdS:Mnalginate nanocomposites was investigated by scanning electron microscopy (SEM) using a FEG SEM Jeol JSM-7600F instrument. Due to the poor conductivity of the materials, the samples were previously coated with gold using a Baltec SCD005 sputter coater.

The morphology of the CdS and CdS:Mn nanoparticles, as well as their dispersion within the alginate matrix were investigated by transmission electron microscopy (TEM) using a Philips Tecnai 20F FEG analytical microscope operating at $200 \mathrm{kV}$. High resolution TEM (HREM) images were acquired for studying the local crystalline quality. For these analyses the materials were grinded and mixed with ethanol, then a single drop of the resulting suspension was deposited on carboncoated copper grids. TEM images were analyzed using Fiji software. $^{39}$

The electron paramagnetic resonance (EPR) experiments were performed on an X-band MagnetTech MS300 spectrometer operating at a nominal frequency of $9.5 \mathrm{GHz}$. The used microwave power was $1 \mathrm{~mW}$ (microwave attenuation $20 \mathrm{~dB}$ ), with a modulation amplitude of $0.2 \mathrm{mT}$.

The UV-vis diffuse reflectance spectroscopy measurements (DR) of the nanocomposites were carried out on a Thermo Evolution 600 spectrophotometer equipped with an integrating sphere diffuse reflectance accessory in the wavelength range from $200 \mathrm{~nm}$ to $900 \mathrm{~nm}$. The UV-vis absorption function $(F(R))$ of the materials was estimated from the reflectance spectra using the Kubelka-Munk equation ${ }^{40}$

$$
F(R)=\frac{1-R^{2}}{2 R}
$$

where $R$ is the reflectance of the sample. The optical band gaps $\left(E_{\mathrm{g}}\right)$ of the nanocomposites were determined using Tauc relation $^{41}$

$$
(F(R) h \nu)^{2}=\text { const. }\left(h \nu-E_{\mathrm{g}}\right)
$$

in which $h \nu$ stands for the energy of the incident light.
The photoluminescence emission and excitation spectra of the CdS-alginate and CdS:Mn-alginate nanocomposites were recorded on a Edinburg, Analytical Instruments CD900 fluorescence spectrophotometer. The spectra were acquired using $10 \mu \mathrm{m}$ slits and $1000 \mathrm{~ms}$ of integration time.

The analytical measurements of the amount of $\mathrm{Cd}^{2+}$ released from the CdS-alginate and CdS:Mn-alginate nanocomposites were performed by atomic absorption spectrometry (AAS) using Perkin Elmer AAnalyst 300 instrument. To perform these measurements, $10 \mathrm{mg}$ of each nanocomposite was placed into clean glass vials containing $10 \mathrm{~mL}$ of sterile saline solution $(0.9 \% \mathrm{NaCl})$. Subsequently, the vials were placed into an oven preheated to $37{ }^{\circ} \mathrm{C}$ and incubated for either one or two hours. Upon incubation, $1 \mathrm{~mL}$ of the solution obtained for each sample was diluted with $9 \mathrm{~mL}$ of saline. Thus obtained solutions were filtered using $0.45 \mu \mathrm{m}$ filter, adjusted to $\mathrm{pH}=2$ using $\mathrm{HNO}_{3}$, and investigated by AAS. It is worth to mention that as a consequence of ionic crosslinking, the nanocomposites were not soluble in saline solution and the nanocomposite powders remained as precipitate at the bottom of the containers. Therefore, only $\mathrm{Cd}^{2+}$ ions released from the material into the saline solution were considered.

The Fourier transform infrared (FTIR) spectroscopy (Model Perkin Elmer Spectrum GX) was employed to study the specific interactions between the semiconductor nanoparticles and the alginate biopolymer in the nanocomposites. The measurements were carried out at room temperature in the $4000-400 \mathrm{~cm}^{-1}$ spectral range with a resolution of $4 \mathrm{~cm}^{-1}$. For these analyses, the nanocomposites were dried in vacuum and $2 \mathrm{mg}$ of the material was mixed with $100 \mathrm{mg}$ of $\mathrm{KBr}$ and pressed into pellets.

The thermogravimetric analysis (TGA) of the materials was performed on a Perkin Elmer STA 6000 device in the temperature interval from $50^{\circ}$ to $800{ }^{\circ} \mathrm{C}$ with a heating rate of $\beta=$

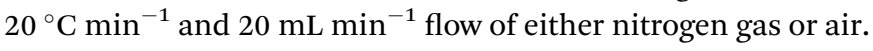

\subsection{Cell toxicity studies of the CdS-alginate and CdS:Mn- alginate nanocomposites}

Human fetal lung fibroblasts MRC-5 (ECACC no. 84101801) cell line was used in this study. The human cells were grown in Dulbecco's modified Eagle's (DMEM) medium containing 4.5\% glucose and $2 \mathrm{mM}$ of L-glutamine, supplemented with heat inactivated $10 \%$ fetal bovine serum, penicillin $\mathrm{G}\left(100 \mathrm{U} \mathrm{mL}^{-1}\right)$ and streptomycin $\left(100 \mu \mathrm{g} \mathrm{mL} \mathrm{m}^{-1}\right)$, at $37{ }^{\circ} \mathrm{C}$ with $5 \% \mathrm{CO}_{2}$ and $100 \%$ humidity. Cell line grows attached to the surface and a single cell suspension was obtained using $0.1 \%$ trypsin. All media, enzymes and supplements for cell cultures were purchased from PAA Laboratories $\mathrm{GmbH}$, Austria.

The CdS-alginate and CdS:Mn-alginate nanocomposites were suspended in DMEM medium (in concentrations 0.5 and $1 \mathrm{mg} \mathrm{mL} \mathrm{m}^{-1}$ ) to allow elution of the material. The samples of each material were used after $1 \mathrm{~h}, 2 \mathrm{~h}$ and $24 \mathrm{~h}$ of elution. The eluates were sterilized by filtration through $0.22 \mu \mathrm{m}$ filters (Sartorius, Germany) and subsequently used for the cytotoxicity testing. The $\mathrm{pH}$ values of the eluates $(1 \mathrm{~h}, 2 \mathrm{~h}$, and $24 \mathrm{~h}$ postelution) were measured on a calibrated Sartorius PB-11 pH meter. The eluates of all tested materials were alkaline $(\mathrm{pH}=8)$ 
for the duration of the experiment and there were no changes in the $\mathrm{pH}$ value over time.

The cytotoxicity of the nanocomposites was determined with the MTT (3-(4,5-dimethylthiazol-2-yl)-2,5-diphenyltetrazolium bromide) reduction assay. MRC-5 cells were seeded onto 96well plates at a density of $3 \times 10^{4}$ cells per well and incubated for $24 \mathrm{~h}$ at $37^{\circ} \mathrm{C}$ to attach. The DMEM medium was then replaced with $200 \mu \mathrm{L}$ of the eluates of each material (6 wells per group). Cells without added material served as a positive control. The cells were incubated for another $24 \mathrm{~h}$ at $37{ }^{\circ} \mathrm{C}$ and $5 \% \mathrm{CO}_{2}$. Subsequently, the MTT (final concentration $0.5 \mathrm{mg} \mathrm{mL}^{-1}$ ) was added and the plates and they were incubated for additional $3 \mathrm{~h}$. At the end of the incubation with MTT, the medium was removed, and the formazan crystals were dissolved in the DMSO. The optical density (OD) of the DMSO solution was measured at $570 \mathrm{~nm}$ (reference filter $690 \mathrm{~nm}$ ) using a Thermo Scientific Multiscan FC microplate reading spectrophotometer. Cell viability was determined by comparing the OD of the wells containing cells treated with the eluates from the nanocomposites to the untreated cells. Six individual wells were measured per treatment point in two independent experiments.

The one-way analysis of variance of the results of the cytotoxic assay (non-parametric ANOVA, Mann-Whitney $U$ test) was performed using Statistica software (StatSoft Statistica Ver. 6.0, USA).

The obtained results were compared to the MTT cytotoxicity test of a bulk CdS powder (99.99\% pure powder, Sigma-Aldrich).

\section{Results and discussion}

\subsection{Characterization of the nanocomposites}

The preparation of CdS-alginate and CdS:Mn-alginate nanocomposites involved transformation of the biopolymer solution into cross-linked network by complexation of divalent cations with $\alpha$-L-guluronic monomers and subsequent formation of the semiconducting nanoparticles within the cross-linked matrix by interaction of sulfide and cadmium ions at elevated temperature. FTIR analyses of the starting biopolymer, $\mathrm{Cd}^{2+}$ crosslinked alginate and CdS-alginate nanocomposite confirmed the interaction between the nanoparticles and the biopolymer (ESI, Fig. S1†). As it was shown by the TGA, the formation of the nanoparticles caused partial elimination of the interactions between the $\mathrm{Cd}^{2+}$ and $\mathrm{COO}^{-}$ions as suggests a lower thermal stability of the CdS-alginate nanocomposite in comparison to Cd-alginate both in inert and oxidative atmosphere (ESI, Fig. S2†).

Structural analysis of synthesized biopolymer nanocomposites was carried out by XRD technique, and the results are presented in Fig. 1. The representative diffraction patterns of CdS-alginate and CdS:Mn (4 mol\%)-alginate samples show maxima at $2 \theta$ angles $26.8^{\circ}, 44.3^{\circ}$ and $52.3^{\circ}$ that correspond to $(1$ 11 ), ( $\left.\begin{array}{lll}2 & 2 & 0\end{array}\right)$, and ( $\left.\begin{array}{lll}3 & 1 & 1\end{array}\right)$ crystallographic planes of a cubic $F \overline{4} 3 \mathrm{~m}$ (sphalerite) CdS lattice (lattice parameter $a=0.5811 \mathrm{~nm}$ ), respectively. ${ }^{42}$ The small contributions at $\sim 38^{\circ}$ and $39.5^{\circ}$ originate from the substrate. The XRD results suggested that the described synthesis procedure lead to the formation of the crystalline CdS phase in the material. The XRD peaks were very

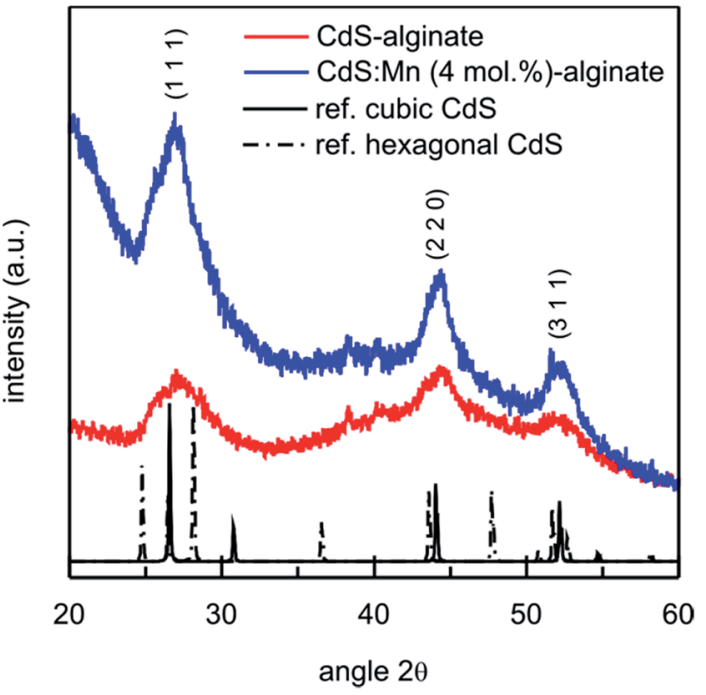

Fig. 1 The XRD patterns of CdS-alginate and CdS:Mn (4 mol\%)-alginate nanocomposites.

broad indicating small crystallite sizes. Using the Scherrer's equation, the average crystallite size of both CdS and CdS:Mn ( $4 \mathrm{~mol} \%$ ) particles was estimated to $3 \mathrm{~nm}$ from the half width of the diffraction peak corresponding to planes $\left(\begin{array}{lll}1 & 1 & 1\end{array}\right)$. The XRD patterns of CdS:Mn (1 mol\%)- and CdS:Mn ( $2 \mathrm{~mol} \%)$-alginate showed similar features as the diffractogram of the nanocomposite with the highest concentration of manganese. It is important to mention that $\mathrm{CdS}$, as well as $\mathrm{Cd}_{1-x} \mathrm{Mn}_{x} \mathrm{~S}$, crystals can also adopt hexagonal (wurtzite) crystalline lattices. ${ }^{42,43}$ In the study on mercaptoethanol capped CdS nanoparticles, the authors showed that both phases coexist in equal proportions in the system. ${ }^{44}$ However, we were unable to estimate the relative contributions of the two phases due to the substantial width of the XRD peaks in the diffraction pattern of the nanocomposite.

SEM micrographs of the grinded CdS-alginate, CdS:Mn (2 mol\%)-alginate, and CdS:Mn (4 mol\%)-alginate samples are presented in Fig. 2. In the case of the CdS-alginate nanocomposite (Fig. 2a), spherical formations approximately $50 \mathrm{~nm}$ in size can be observed on the surface of the sample. Although less pronounced, these structures were also detected in the SEM images of the CdS:Mn-alginate nanocomposites (Fig. 2b and c). We assume that these formations originate from the alginate that was cross-linked in the early stages of the process. More precisely, the formations are the regions with a high ionic crosslinking degree, since the concentration of the divalent cations at time of their creation was the highest. On the other hand, a lower occurrence of the regions with a high degree of the ionic cross-linking in the images of the CdS:Mn-alginate samples suggests that the presence of manganese interferes with the formation of chemical bonds between the $\mathrm{Cd}^{2+}$ ions and the carboxylate groups. Bearing in mind that, regardless of the composition of the biopolymer, Mn has a lower affinity for alginate than $\mathrm{Cd},{ }^{45}$ the observed decrease in the amount of spherical formation with an increase in Mn concentration 

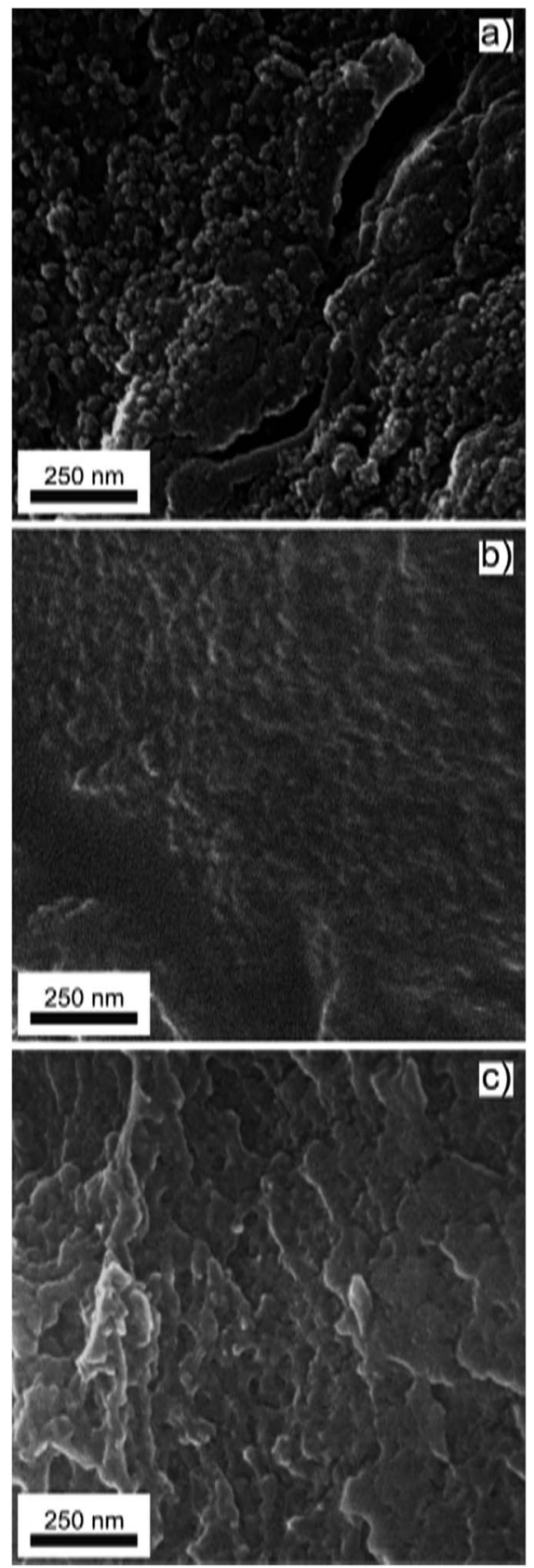

Fig. 2 SEM images of (a) CdS-alginate, (b) CdS:Mn (2 mol\%)-alginate, and (c) CdS:Mn (4 mol\%)-alginate nanocomposite.

further supports our assumption. As it will be seen in the Section 3.3, the differences in the surface morphology of CdSalginate and CdS:Mn-alginate samples result in differences in the $\mathrm{Cd}$ ions release and cell toxicity of these nanocomposites.

Typical TEM images of the CdS-alginate and CdS:Mn (4 mol\%)-alginate nanocomposites are presented in Fig. 3. The micrographs show spherical particles well dispersed within the alginate matrix. The images are weakly out of focus to increase the contrast. The good dispersion of the inorganic phase is a consequence of an in situ approach of the synthesis of the nanostructures, supplemented by the interaction between the
$\mathrm{Cd}^{2+}$ ions and carboxylate groups of the $\alpha$-L-guluronic residues of the biopolymer. In addition, the approach resulted in small particles with narrow size distribution (Fig. 3c). The average diameter of CdS nanoparticles was estimated to $3.6 \pm 0.2 \mathrm{~nm}$, while in the case of the CdS:Mn (4 mol\%)-alginate nanocomposites the corresponding nanostructures were $3.9 \pm$ $0.2 \mathrm{~nm}$ slightly longer than those estimated from the DRX results. TEM images of CdS:Mn (1 mol\%)- and CdS:Mn ( $2 \mathrm{~mol} \%$ )-alginate samples showed negligible differences in size and morphology of the nanoparticles in comparison to CdS:Mn (4 mol\%)-alginate.

High resolution TEM (HRTEM) analyses of an individual nanoparticle in the CdS:Mn (2 mol\%)-alginate sample are presented in Fig. 3d. The image shows lattice fringes oriented in one direction along the whole surface of the nanoparticle indicating that the CdS:Mn nanostructures are composed of single crystals. This was further demonstrated by calculating the Fourier transform of the image (Fig. 3d), which was used to simulate the electron diffraction pattern. The single crystalline structure of the nanoparticle suggests that the final particles synthesized in situ are being formed by nucleation and growth processes rather than the aggregation of the neighboring cluster or particles. This formation mechanism is a consequence of $\mathrm{Cd}^{2+}-\mathrm{COO}^{-}$interaction (ESI, Fig. S1 $\dagger$ ) that must hinder diffusion of the CdS through the matrix. The distances between the crystallographic planes on the nanoparticle surface were calculated by averaging the interplanar separations in a 3 by $1.5 \mathrm{~nm}$ particle area (yellow rectangle on the HRTEM image in Fig. 3d). The average value was $0.205 \pm 0.007 \mathrm{~nm}$ that, with the resolution achieved, corresponds to the distance between two consecutive ( $\left.\begin{array}{lll}2 & 2 & 0\end{array}\right)$ planes in sphalerite CdS. The distance profile of the HRTEM image intensity (gray value) along the direction perpendicular to the (2 20 ) planes is also presented in Fig. 3d. In addition, the profile illustrates irregularities in the periodicity of the fringes that is the consequence of the presence of defects in the crystalline structure of the nanoparticle.

The EPR spectrum of CdS:Mn (4 mol\%)-alginate nanocomposite recorded at room temperature is shown in Fig. 4 . It can be seen that the spectrum consists of a group of six narrow bands superimposed on a broad resonance. The broad signal originates from the smeared contribution due to non-central transitions and the magnetic dipole-dipole interaction of proximate $\mathrm{Mn}^{2+}$ ions, whereas the narrow six bands appear because of the hyperfine interaction between the electron and nuclear spin $(I=5 / 2)$ of $\mathrm{Mn}^{2+}$ ions. The sextet comes from isolated $\mathrm{Mn}^{2+}$ ions incorporated in the CdS lattice since $\mathrm{Mn}-\mathrm{Mn}$ pairs or MnS do not produce the six-line spectrum that we observed. This result suggests that a much lower amount of $\mathrm{Mn}^{2+}$ than $4 \mathrm{~mol} \%$ was incorporated in the lattice, while the residual manganese was removed in the purification steps (see Experimental). Since the hyperfine interactions strongly depend on the local environment of $\mathrm{Mn}^{2+}$ ions, the EPR spectrum can be used to provide more information on the specific location of the ions in the CdS lattice.

More specifically, a six-line pattern with a hyperfine splitting of $A \sim 6.9 \mathrm{mT}$ is typical for $\mathrm{Mn}^{2+}$ located in cation sites with tetrahedral coordination and it is attributed to $\mathrm{Mn}^{2+}$ near the 

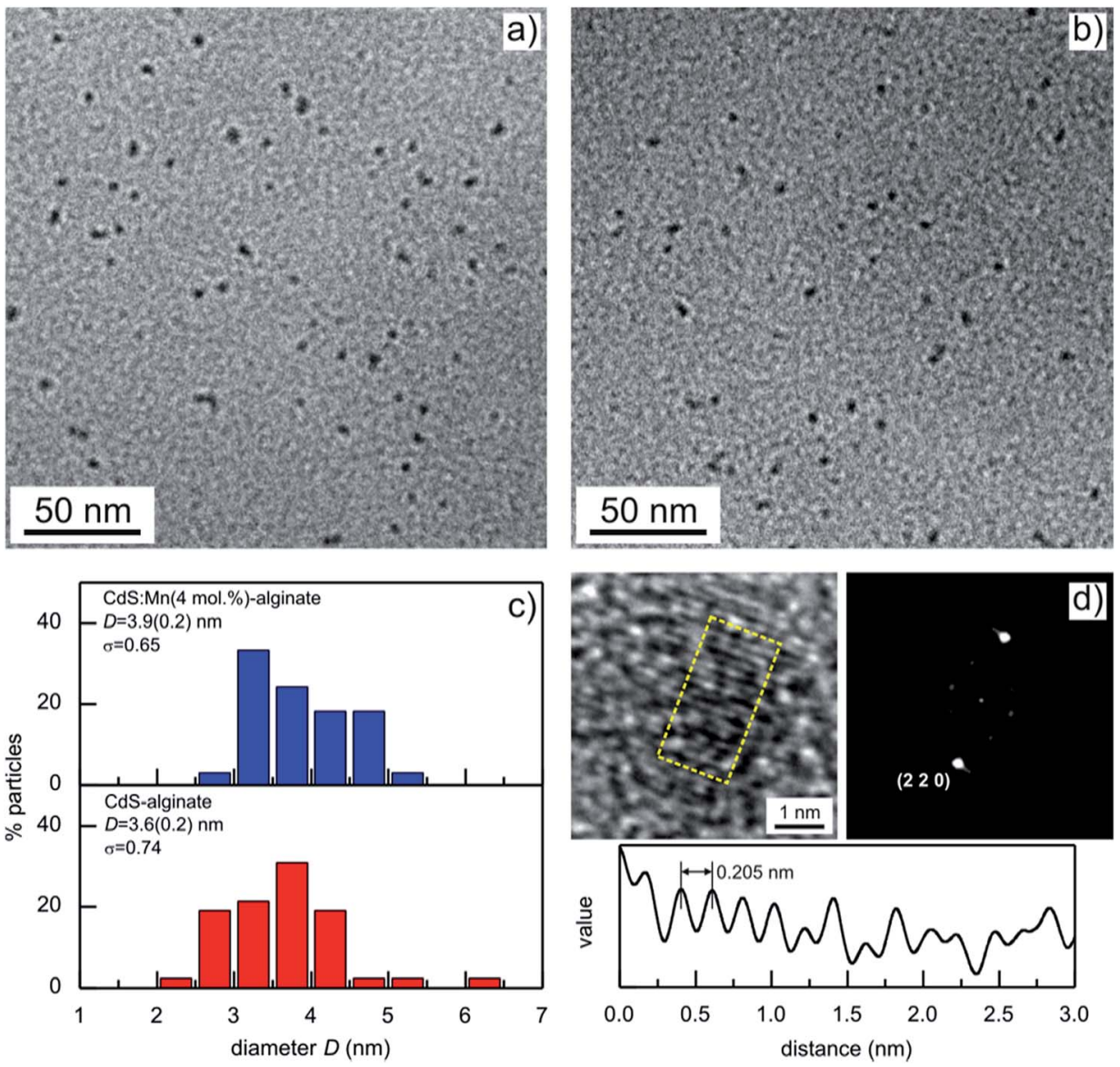

Fig. 3 Bright field TEM images of (a) CdS-alginate and (b) CdS:Mn (4 mol\%)-alginate nanocomposites. (c) Particle size distribution histogram for CdS and CdS:Mn (4 mol\%) particles. (d) HRTEM image a single CdS:Mn (2 mol\%) particle, the corresponding FTP, and the HRTEM image distance profile in $3 \mathrm{~nm} \times 1.5 \mathrm{~nm}$ particle area (the rectangle in the HRTEM image).

center of the nanocrystal. ${ }^{46}$ However, the spectrum of the sample under consideration (Fig. 4.) shows hyperfine structure with hyperfine splitting constant closer to the $A \sim 9.6 \mathrm{mT}$ value that corresponds to the case when $\mathrm{Mn}^{2+}$ ions occupy sites with octahedral coordination. The high $A$ value indicates an increase in the ionic character of the $\mathrm{Mn}^{2+}$ bonding, which is likely to be the case of cations located near the surface of the nanocrystals. $^{47}$ This is somewhat expected result since for spherical CdS nanoparticles of approximately $4 \mathrm{~nm}$ in size, about $64 \%$ of cations are located in the surface shell one lattice constant wide ( $a=0.5811 \mathrm{~nm}$ ), while $93 \%$ of the cations are in the surface region that extends two lattice constants. Similar $A$ values were established for $\mathrm{Mn}^{2+}$ doped CdS nanocrystals (9.6 mT), ${ }^{48}$ ZnS:Mn nanocrystals (9.5 mT), ${ }^{46}$ and in the surface layers of Mndoped CdSe nanocrystals $(8.9 \mathrm{mT}) .^{49}$

To determine contributions to the EPR spectrum of CdS:Mnalginate nanocomposite other than that of $\mathrm{Mn}^{2+}$ ions in the octahedral coordination, we simulated a six line hyperfine spectrum with $A=9.7 \mathrm{mT}$ and $D=0$ (red line in Fig. $4 \mathrm{~b}$ ) and subtracted it from the experimental curve. The difference spectrum is presented by the green line in Fig. $4 \mathrm{~b}$. As it can be seen, it is composed of 5 equidistant peaks with positions in the middle of the corresponding simulated spectrum. Near the surface of the nanoparticle the crystal field symmetry is distorted and the axial fine structure parameter becomes relevant, leading to the relaxation of the $\Delta m_{\mathrm{I}}=0$ selection rule. Consequently, the peaks in the residual spectrum originate from 5 pairs of otherwise forbidden $\Delta m_{\mathrm{I}}= \pm 1$ lines. The intense forbidden transitions are characteristic for polycrystalline and disordered materials, ${ }^{50}$ and reflect distortion of the crystal lattice surrounding $\mathrm{Mn}^{2+}$ ions. As it will be seen in the following section, the fact that $\mathrm{Mn}^{2+}$ ions occupy lattice sites with lower symmetry has significant implications on the photoluminescence spectra of CdS:Mn-alginate nanocomposites.

\subsection{Optical properties of the nanocomposites}

In Fig. 5 UV-vis diffuse reflectance spectra of CdS-alginate and CdS:Mn (4 mol\%)-alginate nanocomposites are shown. Both curves show onsets at approximately $550 \mathrm{~nm}$ that corresponds to the optical electron transitions across the band structure of the semiconductors. Using the Tauc relation (eqn (2)) the optical bandgaps for the materials were estimated to $E_{\mathrm{g}}=2.35$ $\pm 0.08 \mathrm{eV}$ (inset to Fig. 5). Previous reports ${ }^{28,32}$ also gave evidence of the negligible effect of doping by $\mathrm{Mn}^{2+}$ ions in low concentrations on the bandgap of the semiconductors. Obtained values are in agreement with the bandgap of the bulk, ${ }^{24}$ 


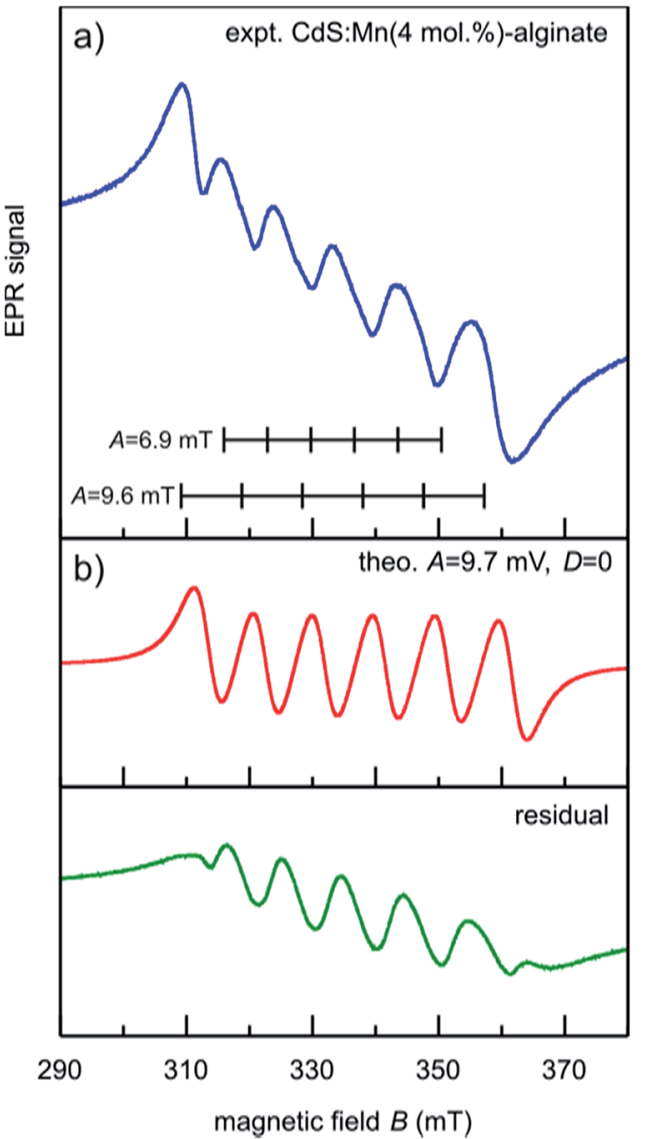

Fig. 4 (a) Experimental EPR spectrum of CdS:Mn (4 mol\%)-alginate nanocomposite. (b) Simulated EPR spectrum for $\mathrm{Mn}^{2+}$ ions in the $\mathrm{CdS}$ lattice for $A=9.7 \mathrm{mT}$ and $D=0$ (red line) and the corresponding difference spectrum (green line).

indicating absence of the exciton confinement in the nanostructures. This result is expected bearing in mind that the sizes of CdS and CdS:Mn nanoparticles exceed the value of the exciton Bohr radius of CdS. In addition, the similar values of the bandgaps found for both materials suggest that the introduction of $\mathrm{Mn}^{2+}$ ions into CdS lattice does not induce additional optically observable defects in the host, otherwise the bandgap of the CdS:Mn nanostructures would decrease.

The photoluminescence emission spectra of CdS:Mn (1 mol\%)-, CdS:Mn (2 mol\%)-, and CdS:Mn (4 mol\%)-alginate nanocomposites obtained for $490 \mathrm{~nm}$ excitation are presented in Fig. 6a. The spectra of the materials show bands with maxima at approximately $570 \mathrm{~nm}(2.17 \mathrm{eV})$, as well as a broad red emission band with maxima at $650 \mathrm{~nm}(1.91 \mathrm{eV}), 667 \mathrm{~nm}$ $(1.86 \mathrm{eV})$, and $690 \mathrm{~nm}(1.80 \mathrm{eV})$ in the case of CdS:Mn (1 mol\%)-, CdS:Mn (2 mol\%)-, and CdS:Mn (4 mol\%)-alginate nanocomposite, respectively. For comparison, the spectrum of CdSalginate nanocomposite is also presented. The high energy band corresponds to exciton emission due to band to band electronic transitions in the nanostructured semiconductor, while the red photoluminescence originates from combined effects of the radiative transitions from the recombination of charge carriers via defect or surface states and the $\mathrm{Mn}^{2+}$ energy $(\mathrm{eV})$

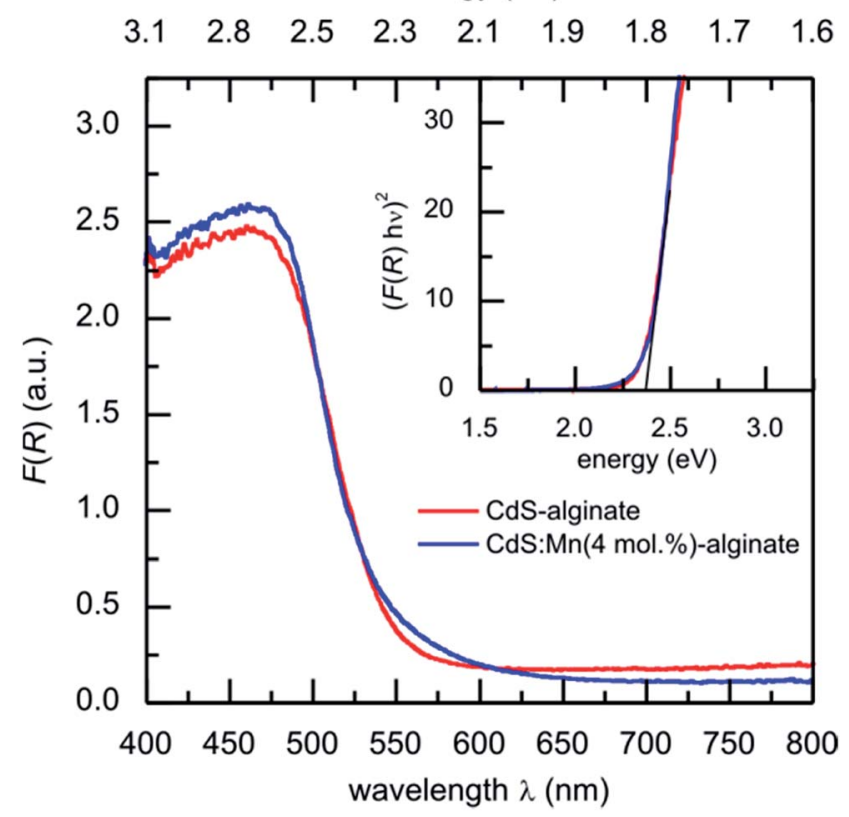

Fig. 5 UV-vis diffuse reflectance spectra $F(R)$ curves of CdS-alginate (red line) and CdS:Mn (4 mol\%)-alginate (blue line) nanocomposites. Inset: Tauc graph corresponding to $F(R)$ for both materials.

photoemission. The described photoluminescence of the nanocomposites was consistent with the change in the excitation wavelength between $470 \mathrm{~nm}$ and $500 \mathrm{~nm}$ (Fig. 5b).

As it was mentioned previously, $\mathrm{Mn}^{2+}$ emission originates from localized 3d states that are strongly affected by the symmetry of the crystal field of the lattice site occupied by the ion. It was not possible to unambiguously resolve the yellow emission $(585 \mathrm{~nm})$ in the photoluminescence spectra of CdS:Mn nanocomposites that originates from the ${ }^{4} \mathrm{~T}_{1}$ to ${ }^{6} \mathrm{~A}_{1}$ transitions of $\mathrm{Mn}^{2+}$ ions positioned in sites with tetrahedral coordination. Consequently, the observed red emission of the nanocomposites, which, in addition is concentration dependent, indicates different local environments of the dopant. The red emission of $\mathrm{Mn}^{2+}$ in II-VI semiconductors was reported previously for hollow $\mathrm{Cd}_{1-x} \mathrm{Mn}_{x} \mathrm{~S}$ quantum wires, ${ }^{31}$ 1-thioglycerol caped $\mathrm{Mn}^{2+}$ doped CdS nanoparticles, ${ }^{28,34}$ as well as on individual nanocrystals of ZnS-CdS alloys doped with $\mathrm{Mn} .{ }^{35} \mathrm{In}$ their combined experimental and ab initio theoretical study, Nag et al. ${ }^{34}$ showed that the red emission comes from the $3 \mathrm{~d}-$ internal transition of $\mathrm{Mn}^{2+}$ ions located near the surface regions of the CdS host that are influenced by crystal fields of distorted tetrahedral symmetry. The EPR results of the present work are in accordance with that study. In addition, Chen et al. ${ }^{51}$ reported that the yellow $\mathrm{Mn}^{2+}$ emission can be red-shifted by applying pressure to $\mathrm{ZnS:} \mathrm{Mn}^{2+}$ nanoparticles.

Therefore, due to limited diffusion of the $\mathrm{Cd}^{2+}$ and $\mathrm{Mn}^{2+}$ cations within the alginate matrix, $\mathrm{Mn}^{2+}$ ions are able to occupy only sites near the surface of CdS nanoparticles of lower than tetrahedral symmetry that leads to the observed red emission of the CdS:Mn-alginate nanocomposites. In addition, with an increase in $\mathrm{Mn}^{2+}$ concentration, the number of occupied sites 

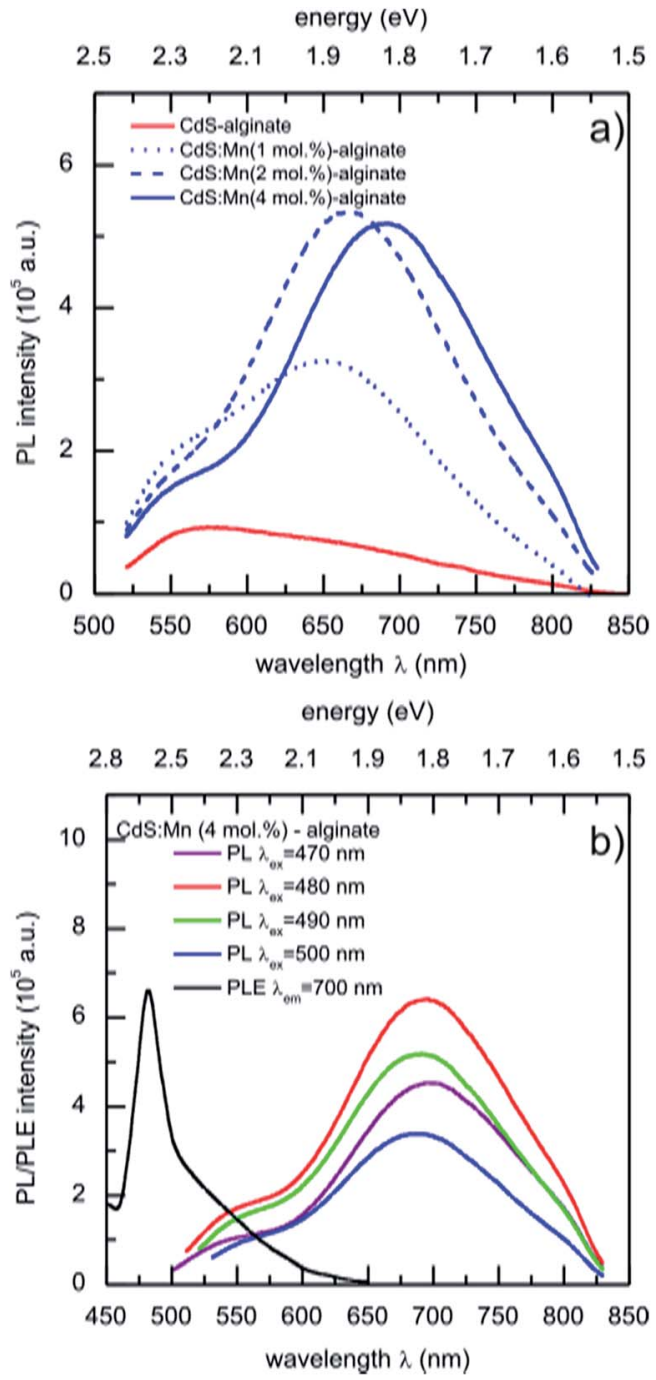

Fig. 6 (a) Photoluminescence emission spectra of CdS-alginate and CdS:Mn-alginate nanocomposites ( $\lambda_{\text {exc }}=490 \mathrm{~nm}$ ). (b) Photoluminescence emission (PL) and excitation (PLE) spectra of CdS:Mn (4 mol\%)-alginate nanocomposite.

with lower symmetry also increases, leading to the red shift of the red-emission band. It is worth to mention that the photoluminescence of the CdS:Mn-alginate nanocomposites falls into the near-infrared window useful for bioimaging and then opening the possibility of using this nanomaterials for tissue imaging applications. ${ }^{5}$

\subsection{Cytotoxicity of CdS-alginate and CdS:Mn-alginate nanocomposites}

Due to the inherent acute cytotoxicity of Cd-based quantum dots, ${ }^{52} \mathrm{CdS}: \mathrm{Mn}$-alginate nanocomposites should be tested using MTT assay in order to see if they are suitable materials for the fluorescence bioimaging applications. The toxicity of CdS quantum dots is a consequence two factors. First factor is the toxicity of cadmium, which is well established and relies on an increase in oxidative stress, since this heavy metal acts as a catalyst in the formation of reactive oxygen species. ${ }^{53}$ The second factor is related to the small dimensions of quantum dots that results in their facile diffusion through tissues and their possible accumulation in the intracellular regions. ${ }^{54}$ Manganese, on the other hand, is an essential trace element required for enzymatic reactions. A recent study, however, reported that continuous exposure to this element may promote neurodegenerative damage. ${ }^{55}$ It was previously reported that coating of quantum dots by an appropriate surface layers reduces their toxicity (see for example ref. 52 and references therein). Consequently, we assume that the cytotoxicity of CdSalginate and CdS:Mn-alginate nanocomposites would be low, since both the diffusion of the nanoparticles and the leaching of cadmium from the nanomaterial is inhibited by the interaction between the $\mathrm{Cd}^{2+}$ ions and the $\alpha$-L-guluronic monomers of alginate.

The amount of cadmium released from the $1.0 \mathrm{wt} / \mathrm{vol} \%$ saline dispersion of CdS-alginate and CdS:Mn (4 mol\%)-alginate nanocomposite, after $1 \mathrm{~h}$ and $2 \mathrm{~h}$ of leaching at $37^{\circ} \mathrm{C}$ was measured by the AAS. The results are presented in Table 1 . In the case of CdS-alginate sample, the detected concentration of $\mathrm{Cd}^{2+}$ did not exceed $1.5 \mathrm{ppm}$, while the amount of $\mathrm{Cd}^{2+}$ released from the CdS:Mn (4 mol\%)-alginate nanocomposite was approximately 6 times higher. It is important to note that the initial concentration of $\mathrm{Cd}^{2+}$ in both samples was the same. The reason behind the difference in the leaching of cadmium between two samples arises from the different surface morphologies (Fig. 2). More specifically, in the CdS-alginate nanocomposite the $\mathrm{Cd}^{2+}$ ions are chemically bound to the $\alpha-\mathrm{L}-$ guluronic monomers and form spherical structures of the biopolymer in the initial stages of the ionic cross-linking. On the other hand, in the CdS:Mn-alginate nanocomposite, due to competitive ion exchange with $\mathrm{Mn}^{2+}$ part of the $\mathrm{Cd}^{2+}$ ions might remain in the vicinity of the biopolymer surface. This assumption can be supported by noting that the majority of $\mathrm{Cd}^{2+}$ was immediately released, since the additional hour of leaching from the CdS:Mn (4 mol\%)-alginate nanocomposite resulted in an increase of cadmium concentration by less than $5 \%$.

The results of the toxicity of CdS-alginate and CdS:Mn (4 mol\%)-alginate nanocomposites versus human fetal lung fibroblasts MRC- 5 cells obtained using MTT assay are presented in Fig. 7. The tests were performed using eluates obtained after $1 \mathrm{~h}, 2 \mathrm{~h}$, and $24 \mathrm{~h}$ of elution of the nanocomposite dispersions prepared in high concentrations ( $0.5 \mathrm{wt} / \mathrm{vol} \%$ and $1.0 \mathrm{wt} / \mathrm{vol} \%$ ). It can be seen that the metabolic activity of MRC- 5 cell line was not significantly affected by a $24 \mathrm{~h}$ of treatment with the eluates of the nanocomposites obtained after $1 \mathrm{~h}$ of elution. The reduction in viable cells to $87 \%$, was measured for the eluate of the $1.0 \mathrm{wt} / \mathrm{vol} \% \mathrm{CdS}: \mathrm{Mn}$ (4 mol\%)-alginate DMEM dispersion,

Table 1 The amount of cadmium released from the $1.0 \mathrm{wt} / \mathrm{vol} \%$ saline dispersion of CdS-alginate and CdS:Mn (4 mol\%)-alginate nanocomposites

\begin{tabular}{lll}
\hline Time & CdS-alginate & CdS:Mn (4 mol\%)-alginate \\
\hline $1 \mathrm{~h}$ & $1.4 \mathrm{ppm}$ & $8.5 \mathrm{ppm}$ \\
$2 \mathrm{~h}$ & $1.5 \mathrm{ppm}$ & $8.9 \mathrm{ppm}$
\end{tabular}




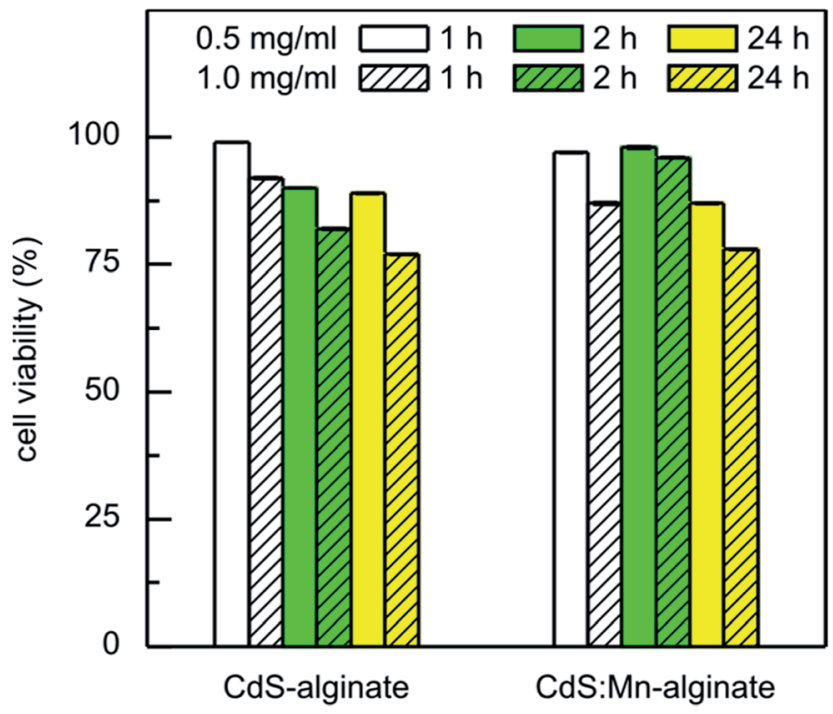

Fig. 7 Influence of CdS-alginate and CdS:Mn (4 mol\%)-alginate nanocomposites on the viability of human fetal lung fibroblasts MRC-5 cells determined by MTT assay. The cells were exposed for $24 \mathrm{~h}$ to the eluates of CdS-alginate and CdS:Mn (4 mol\%)-alginate nanocomposite DMEM dispersion (concentrations $0.5 \mathrm{wt} / \mathrm{vol} \%$ and $1.0 \mathrm{wt} /$ vol\%) obtained after $1 \mathrm{~h}, 2 \mathrm{~h}$, and $24 \mathrm{~h}$ of elution.

while $92 \%$ of the cells survived in the case of the dispersion of CdS-alginate of the same concentration. As it was shown by AAS, the higher toxicity of the material containing Mn-doped CdS nanoparticles is a consequence of a larger amount of $\mathrm{Cd}$ that was released from this nanocomposite.

With an increase in the elution time to $2 \mathrm{~h}$ and $24 \mathrm{~h}$, the mortality of the cells generally increased. However, the eluates of the CdS:Mn (4 mol\%)-alginate dispersion obtained after $2 \mathrm{~h}$ of elution showed lower cytotoxicity (cell viability was $98 \%$ and $96 \%$ for the 0.5 and $1.0 \mathrm{wt} / \mathrm{vol} \%$ concentration, respectively), probably due to a depletion of cadmium from the surface regions of the nanocomposite. Therefore, since cadmium induces acute cytotoxicity, upon depletion of the heavy metal from the surface of the CdS:Mn (4 mol\%)-alginate nanocomposite the MRC-5 cells recuperate and recover their normal metabolic activity. The lowest percentage of cell survival for all investigated cases was $77 \%$, measured for the eluates of the $1.0 \mathrm{wt} / \mathrm{vol} \% \mathrm{CdS}$-alginate dispersion after $24 \mathrm{~h}$ of elution time.

Concentration dependent cytotoxicity of bare CdS nanoparticles, $5-7 \mathrm{~nm}$ in size, determined by MTT assay was reported previously. ${ }^{56}$ The study showed that the metabolic activity of CHL cells reduced from $84 \%$, upon exposure to $2.5 \mathrm{ppm}$ of the particles, to about $20 \%$ when the cells were treated with CdS quantum dots of concentrations ranging from 20 to $80 \mathrm{ppm}$. In our case, the nominal concentration of the nanoparticles in the nanocomposite was $10 \mathrm{wt} \%$ that leads to a total CdS concentration in the $1.0 \mathrm{wt} / \mathrm{vol} \%$ nanocomposite dispersion of approximately $100 \mathrm{ppm}$. However, as a consequence of the chemical structure of the biopolymer and chemical bonding between $\mathrm{Cd}^{2+}$ and $\alpha$-L-guluronic monomers, the nanoparticle diffusion from the matrix into the environment is inhibited and the amount of released cadmium is reduced significantly (Table
1). Consequently, the MRC-5 cells were exposed to CdS in a much lesser concentrations, which resulted in the low cytotoxicity of CdS-alginate and CdS:Mn-alginate nanocomposites.

\section{Conclusions}

In this study, we employed biocompatible Na-alginate biopolymer as a template for an in situ chemical synthesis of manganese-doped cadmium sulfide nanocrystals in aqueous solution. The results of the structural investigations showed that the dopant was distributed in octahedral sites near the surface of nanocrystal and suggested a distortion of the crystal lattice surrounding $\mathrm{Mn}^{2+}$ ions. This preferential positioning in the sites of lower symmetry resulted in the photoluminescence of the nanocomposites between $650 \mathrm{~nm}$ and $750 \mathrm{~nm}$. Because of the chemical bonding between $\mathrm{Cd}^{2+}$ ions and alginate, the cytotoxicity of the prepared nanocomposites, was low in comparison to the data reported for bare CdS nanoparticles.

Few additional remarks are worth to mention regarding the study on CdS:Mn-alginate nanocomposites. First, the presented synthesis results in high concentration of the nanoparticles with respect to the polymer and can be easily scaled to produce larger amounts of the nanocomposite. Also, the general procedure can be used for the preparation of alginate nanocomposites with a variety of transition metal doped II-VI semiconductor nanoparticles (ZnS:Mn, CdS:Cu, etc.). Furthermore, by using water soluble precursors and the biopolymer in the synthesis, we have avoided the use of toxic capping agents and organic solvents that are typically employed for the preparation of semiconductor nanoparticles. Finally, low cost redemitting CdS:Mn-alginate nanocomposites of low toxicity could be advantageous for future fluorescence bioimaging studies.

\section{Conflicts of interest}

There are no conflicts to declare.

\section{Acknowledgements}

This research was financially supported by the Ministerio de Ciencia e Innovación (grant MAT2010-16815); Ministerio de Economía y Competitividad (grant MAT2014-59116-C2); Fondos de Investigación de Fco. Javier Gonzalez Benito, política de reinversión de costes generales, Universidad Carlos III de Madrid (Ref.: 2012/00130/004); Acción Estratégica en Materiales Compuestos Poliméricos e Interfases, Universidad Carlos III de Madrid (Ref.: 2011/00287/002), and TECHNOFUSION(II)-CM (S2013/MAE-2745) Spain. M. K. acknowledges University Carlos III Madrid for the financial support via 'Ayudas al studio de Master Universitario'. D. K. B. would like to thank the University Carlos III Madrid for the 'Estancias postdoctorales fellowship'. TEM characterization was made at LABMET, associated to the Red de Laboratorios de la Comunidad de Madrid. D. K. B and D. $\mathrm{M}$ acknowledge the Ministry of Education and Science of the Republic of Serbia for the financial support (Project No. 172056 and 45020). 


\section{References}

1 A. C. Balazs, T. Emrick and T. P. Russell, Science, 2006, 314, 1107.

2 P. K. Sudeep and T. Emrick, Polym. Rev., 2007, 47, 155.

3 W. U. Huynh, J. J. Dittmer and A. P. Alivisatos, Science, 2002, $295,2425$.

4 D. Y. Godovsky, Nanocomposites, 2000, 153, 163.

5 J. M. Klostranec and W. C. W. Chan, Adv. Mater., 2006, 18, 1953.

6 I. Sondi, A. Siiman and E. Matijevic, J. Colloid Interface Sci., 2004, 275, 503.

7 Q. L. Nie, W. B. Tan and Y. Zhang, Nanotechnology, 2006, 17, 140.

8 T. Radhakrishnan, M. K. Georges, P. S. Nair, A. S. Luyt and V. Djokovic, J. Nanosci. Nanotechnol., 2007, 7, 986.

9 P. Rodriguez, N. Munoz-Aguirre, E. S. M. Martinez, G. G. de la Cruz, S. A. Tomas and O. Z. Angel, J. Cryst. Growth, 2008, 310, 160.

10 N. Vigneshwaran, S. Kumar, A. A. Kathe, P. V. Varadarajan and V. Prasad, Nanotechnology, 2006, 17, 5087.

11 D. K. Božanić, V. Djoković, J. Blanuša, P. S. Nair, M. K. Georges and T. Radhakrishnan, Eur. Phys. J. E: Soft Matter Biol. Phys., 2007, 22, 51.

12 D. K. Božanić, V. Djoković, N. Bibić, P. S. Nair, M. K. Georges and T. Radhakrishnan, Carbohydr. Res., 2009, 344, 2383.

13 V. Djoković, R. Krsmanović, D. K. Božanić, M. McPherson, G. Van Tendeloo, P. S. Nair, M. K. Georges and T. Radhakrishnan, Colloids Surf., B, 2009, 73, 30.

14 W. T. Wu, M. Aiello, T. Zhou, A. Berliner, P. Banerjee and S. Q. Zhou, Biomaterials, 2010, 31, 3023.

15 S. K. Chanda, L. Hirst, E. G. V. Percival and A. G. Ross, J. Chem. Soc., 1952, 1933.

16 H. H. Zheng, Carbohydr. Res., 1997, 302, 97.

17 C. H. Wang, Y. S. Hsu and C. A. Peng, Biosens. Bioelectron., 2008, 24, 1012.

18 P. Sundarrajan, P. Eswaran, A. Marimuthu, L. B. Subhadra and P. Kannaiyan, Bull. Korean Chem. Soc., 2012, 33, 3218.

19 R. Brayner, M. J. Vaulay, F. Fievet and T. Coradin, Chem. Mater., 2007, 19, 1190.

20 H.-L. Ma, X.-R. Qi, Y. Maitani and T. Nagai, Int. J. Pharm., 2007, 333, 177.

21 L. V. Trandafilovic, D. K. Bozanic, S. Dimitrijevic-Brankovic, A. S. Luyt and V. Djokovic, Carbohydr. Polym., 2012, 88, 263.

22 L. V. Trandafilović, R. Krsmanović Whiffen, S. DimitrijevićBranković, M. Stoiljković, A. S. Luyt and V. Djoković, Chem. Eng. J., 2014, 253, 341.

23 A. M. Laera, V. Resta, E. Piscopiello, V. Miceli, M. Schioppa, A. Scalone, G. F. Di Benedetto and L. Tapfer, Nanoscale Res. Lett., 2013, 8, 382.

24 J. Wang and M. Isshiki, Springer Handbook of Electronic and Photonic Materials, 2007.

25 W. S. Chae, J. H. Ko, I. W. Hwang and Y. R. Kim, Chem. Phys. Lett., 2000, 365, 49.

26 M. Tanaka, J. Lumin., 2002, 100, 163.
27 S. M. Liu, F. Q. Liu, H. Q. Guo, Z. H. Zhang and Z. G. Wang, Solid State Commun., 2000, 115, 615.

28 A. Nag, S. Sapra, C. Nagamani, A. Sharma, N. Pradhan, S. V. Bhat and D. D. Sarma, Chem. Mater., 2007, 19, 3252.

29 P. Wu and X. P. Yan, Chem. Soc. Rev., 2013, 42, 5489.

30 W. Chen, R. Sammynaiken, Y. N. Huang, J. O. Malm, R. Wallenberg, J. O. Bovin, V. Zwiller and N. A. Kotov, J. Appl. Phys., 2001, 89, 1120.

31 F. J. Brieler, M. Froba, L. M. Chen, P. J. Klar, W. Heimbrodt, H. A. K. von Nidda and A. Loidl, Chem.-Eur. J., 2002, 8, 185.

32 R. M. Krsmanović Whiffen, D. J. Jovanovic, Z. Antić, B. Bartova, D. Milivojevic, M. D. Dramicanin and M. G. Brik, J. Lumin., 2014, 146, 133.

33 L. L. Wang, L. L. Tan, T. Hou and J. S. Shi, J. Lumin., 2013, 134, 319.

34 A. Nag, R. Cherian, P. Mahadevan, A. V. Gopal, A. Hazarika, A. Mohan, A. S. Vengurlekar and D. D. Sarma, J. Phys. Chem. $C, 2010,114,18323$.

35 A. Hazarika, A. Layek, S. De, A. Nag, S. Debnath, P. Mahadevan, A. Chowdhury and D. D. Sarma, Phys. Rev. Lett., 2013, 110, 5.

36 R. Beaulac, P. I. Archer and D. R. Gamelin, J. Solid State Chem., 2008, 181, 1582.

37 Y. R. Huang, S. He, W. P. Cao, K. Y. Cai and X. J. Liang, Nanoscale, 2012, 4, 6135.

38 V. J. Pansare, S. Hejazi, W. J. Faenza and R. K. Prudhomme, Chem. Mater., 2012, 24, 812.

39 J. Schindelin, I. Arganda-Carreras, E. Frise, V. Kaynig, M. Longair, T. Pietzsch, S. Preibisch, C. Rueden, S. Saalfeld, B. Schmid, J. Y. Tinevez, D. J. White, V. Hartenstein, K. Eliceiri, P. Tomancak and A. Cardona, Nat. Methods, 2012, 9, 676.

40 P. Kubelka and F. Munk, J. Tech. Phys., 1931, 12, 593.

41 J. Tauc, Mater. Res. Bull., 1968, 3, 37.

42 C. Y. Yeh, Z. W. Lu, S. Froyen and A. Zunger, Phys. Rev. B, 1992, 46, 10086.

43 D. Rodic, V. Spasojevic, A. Bajorek and P. Onnerud, J. Magn. Magn. Mater., 1996, 152, 159.

44 W. Vogel, J. Urban, M. Kundu and S. K. Kulkarni, Langmuir, 1997, 13, 827.

45 A. Haug, Acta Chem. Scand., 1961, 15, 1794.

46 T. A. Kennedy, E. R. Glaser, P. B. Klein and R. N. Bhargrava, Phys. Rev. B, 1995, 52, R14356.

47 E. Simanek and K. A. Müller, J. Phys. Chem. Solids, 1970, 31, 1027.

48 G. Counio, S. Esnouf, T. Gacoin and J. P. Boilot, J. Phys. Chem., 1996, 100, 20021.

49 F. V. Mikulec, M. Kuno, M. Bennati, D. A. Hall, R. G. Griffin and M. G. Bawendi, J. Am. Chem. Soc., 2000, 122, 2532.

50 H. W. Wijn and R. F. van Balderen, J. Chem. Phys., 1967, 46, 1381.

51 W. Chen, G. H. Li, J. O. Malm, Y. N. Huang, R. Wallenberg, H. X. Han, Z. P. Wang and J. O. Bovin, J. Lumin., 2000, 91, 139. 
52 V. Kumar, A. Kumari, P. Guleria and S. K. Yadav, Rev. Environ. Contam. Toxicol., 2011, 215, 39.

53 A. Navas-Acien, E. Selvin, A. R. Sharrett, E. CalderonAranda, E. Silbergeld and E. Guallar, Circulation, 2004, 109, 3196.
54 B. L'Azou, I. Passagne, S. Mounicou, M. Treguer-Delapierre, I. Puljalte, J. Szpunar, R. Lobinski and C. Ohayon-Courtes, Toxicol. Res., 2014, 3, 32.

55 J. Crossgrove and W. Zheng, NMR Biomed., 2004, 17, 544.

56 K. G. Li, J. T. Chen, S. S. Bai, X. Wen, S. Y. Song, Q. Yu, J. Li and Y. Q. Wang, Toxicol. in Vitro, 2009, 23, 1007. 